\title{
Developmental Arsenic Neurotoxicity in Retrospect
}

\author{
Philippe Grandjean* and Katsuyuki Murata†
}

Developmental neurotoxicity is of crucial public health importance. The vulnerability of the brain originates from the combination of immaturity and ongoing development; the damage incurred is likely to be permanent. However, epidemiologic studies in this field must confront some serious challenges. First of all, the functional deficits are likely to depend on the developmental stage at which the exposure or the peak exposure occurred. Furthermore, neurobehavioral outcomes will be affected by the age at examination and many other covariates. For example, nutritional deficiencies may cause serious delays in mental development. ${ }^{1}$ The neurotoxicity literature on lead ${ }^{2}$ and methylmercury ${ }^{3}$ contains ample demonstration that neurobehavioral responses vary among populations and may be blurred by the effects of the covariates.

Causative exposures may occur during gestation or soon after birth, whereas valid assessment of cognitive functions may require testing at school age several years later. Thus, the key study parameters are often separated by a substantial time interval. Still, for feasibility reasons, most epidemiologic studies are cross-sectional with some form of retrospective exposure assessment. Estimates of past exposures from questionnaires and residence data are bound to be imprecise and generally tend to bias the findings toward the null. Although the amount of imprecision is unknown, it will likely exceed the substantial variability documented for exposure biomarkers, ie, contaminant concentrations in body fluids and tissues. ${ }^{4}$

The article by von Ehrenstein et $a l^{5}$ in this issue presents a valiant attempt to combine measurements of arsenic in water with residence information to obtain a record of past arsenic exposures in Bengali children. Although this effort failed to demonstrate impact of previous arsenic exposure on measures of school-age cognitive function, the authors demonstrate that information on past arsenic exposure may be obtained and applied in epidemiologic studies.

The authors chose to split the exposures into wide groupings according to regulatory limits. Perhaps some different classification could have provided better separation of exposures. In contrast, current exposure was based on both water intake and urine-arsenic concentrations and was therefore likely to be more precise. Unexpectedly, the authors found that current exposure at age 5 to 15 years was associated with a cognitive deficit, whereas exposure during gestation was not. This could be due to different degrees of imprecision of the exposure variables. This caveat is recognized by the authors and needs emphasis, because the impact of confounders measured with better precision (such as age) may increase the bias toward the null. ${ }^{6}$

In addition to the level of arsenic exposure (in this case, the concentration in drinking water), the timing of exposure deserves careful consideration. Arsenic passes the placental barrier, but transfer through human milk seems to be limited. ${ }^{7}$ The infant may therefore be relatively protected against environmental arsenic exposure during the breast-feeding period. Future studies should, if possible, take into account the time when postnatal water exposure began after the cessation of breast-feeding or the time of introduction of supplementary foods.

As von Ehrenstein et al ${ }^{5}$ note, several recent cross-sectional studies have reported links between arsenic exposures and neurobehavioral deficits in school children. This evidence supports the notion that arsenic is a developmental neurotoxicant. More substantial support derives from evidence of severe clinical effects caused by arsenic contamination of milk powder used for preparation of milk substitute for infants. The reports on this tragedy appeared in Japanese language journals and have only been recently reviewed in English. ${ }^{8}$ Records show that the 
prepared milk contained arsenic concentrations of $2 \mathrm{mg} / \mathrm{L}$ or more. Clinical poisoning occurred after total doses of approximately $60 \mathrm{mg}$ within approximately 1 month. Limited follow-up of the children exposed to contaminated milk powder revealed neurologic diseases, neurobehavioral dysfunction, and decreased cognitive skills. ${ }^{8}$

Judging from the Japanese study, some neurotoxicity would likely be present among the Bengali children at the time of examination, at least among those with the highest exposure levels. Furthermore, if the evidence on lead and methylmercury is of any guidance in regard to arsenic neurotoxicity, subclinical effects might occur even at exposure levels that are 1/100 of the doses that cause clinical poisoning. Accordingly, developmental arsenic exposure within the ranges studied by von Ehrenstein et al could be associated with adverse neurobehavioral effects, although not detected in this study. Given the current information on arsenic neurotoxicity, the absence of significant associations in the Bengali study should therefore not be taken as evidence of safety of these arsenic exposure levels.

The issue of developmental neurotoxicity has been ignored in previous risk assessments of environmental arsenic exposure. Thus, cancer risk has been the basis for current exposure limits, ${ }^{9,10}$ whereas developmental neurotoxicity has not been considered at all. It would seem unwise to overlook arsenic as a likely developmental neurotoxicant. This issue should be an important priority in environmental epidemiology research.

\section{REFERENCES}

1. Chang SM, Walker SP, Grantham-McGregor S, et al. Early childhood stunting and later behaviour and school achievement. J Child Psychol Psychiatry. 2002; 43: 775-783.

2. Bellinger DC. Lead. Pediatrics. 2004; 113 (suppl): 1016 -1022.

3. Grandjean P, Cordier S, Kjellström T. Developmental neurotoxicity associated with dietary exposure to methylmercury from seafood and freshwater fish. In: Bellinger D, ed. Human Developmental Neurotoxicology. New York: Marcel Dekker; 2006: 25- 42.

4. Grandjean P, Budtz-Jørgensen E, Keiding N, et al. Underestimation of risk due to exposure misclassification. Int J Occup Med Environ Health. 2004; 17: 131-136.

5. von Ehrenstein O, Poddar S, Yuan Y, et al. Children's intellectual function in relation to arsenic exposure. Epidemiology 2007; 18: 44-51.

6. Budtz-Jørgensen E, Keiding N, Grandjean P, et al. Consequences of exposure measurement error for confounder identification in environmental epidemiology. Stat Med. 2003; 22: 3089-100.

7. Concha G, Vogler G, Lezcano D, et al. Exposure to inorganic arsenic metabolites during early human development. Toxicol Sci. 1998; 44: 185-190.

8. Dakeishi M, Murata K, Grandjean P. Lessons from arsenic poisoning of infants due to contaminated dried milk: a review. Environ Health. 2006; 5: 31.

9. National Academy of Sciences. Arsenic in Drinking Water. Washington, DC: National Academy Press; 1999.

10. International Programme on Chemical Safety. Arsenic and Arsenic Compounds, 2nd edition (Environmental Health Criteria 224). Geneva: World Health Organization; 2001. 